\title{
Novel Magnetic Properties of Carbon Nanotubes
}

\author{
Jian Ping Lu \\ Department of Physics and Astronomy, University of North Carolina at Chapel Hill, Chapel Hill, \\ North Carolina 27599 \\ jpl@physics.unc.edu
}

(Received November 9, 2018)

\begin{abstract}
An external magnetic field is found to have strong effects on the electronic structure of carbon nanotubes. A field-induced metal-insulator transition is predicted for all pure nanotubes. In a weak field, nanotubes exhibit both large diamagnetic and paramagnetic responses which depend on the field direction, the position of the Fermi energy, the helicity, and the size of the nanotube. Universal scalings are found for the susceptibility as functions of the Fermi energy, the temperature, and the size of nanotubes. These results are in agreement with experiments.
\end{abstract}

PACS numbers: $61.46+\mathrm{w}, 36.40+\mathrm{d}$ 
The exciting discovery of carbon nanotubes [四] stimulated a large number of theoretical studies on their electronic properties. Both tight-binding [2, 3] and first principles calculations [U] predicted that nanotubes can be either metallic or semiconducting depending on their helicity and size. Several recent experiments demonstrated some unusual properties. Giant magnetoresistance and indications of a field-induced metal-insulator transition are found in transport measurements [5]. Large diamagnetic susceptibilities are found for a magnetic field both perpendicular and parallel to the tube axis [6]:7].

The magnetic properties of nanotubes were studied by Ajiki and Ando [8] using the $\mathbf{k} \cdot \mathbf{p}$ perturbation method However, they found that the susceptibility $\chi_{\perp}$ (for the field $\mathbf{H}$ perpendicular to the tube axis $\hat{\mathbf{z}})$ is three orders of magnitude larger than $\chi_{\|}($when $\mathbf{H} \| \hat{\mathbf{z}})$. This finding disagrees with experiments, where it is found that the two are comparable [6]. The $\mathbf{k} \cdot \mathbf{p}$ calculation is valid only if the Fermi energy is at the center of the band (half filling), and it only provides information about the band structure near the Fermi energy. The orbital magnetism depends on the total band energy which requires the calculation of the total band structure. In addition, in real materials it is likely that the system is not exactly at the half filling (corresponds to the case of a finite carrier density in graphite). Thus, a calculation which includes the field dependence of the complete $\pi$ band is necessary to understand the magnetic response of the nanotube.

In this letter we report the results of such a calculation using the tight-binding model and the London approximation. We obtained the following results. 1) A magnetic field induced metal-insulator transition is predicted for all pure nanotubes, the transition depends on the helicity, the radius $R$, and the magnetic field direction. 2) The weak-field magnetic susceptibility is large and increases linearly with the size of nanotubes, $\chi \sim R$; it can be either diamagnetic or paramagnetic and is sensitive to the position of the Fermi energy $\epsilon_{F}$. 3) Associate with each nanotube is a unique energy scale $\Delta_{0}$; the scaled susceptibility $\chi / R$ is found to be a universal function of $\epsilon_{F} / \Delta_{0}$ and $k_{B} T / \Delta_{0}$ for each family of nanotubes. 4) For typical nanotubes, $\chi \sim-250 \times 10^{-6} \operatorname{cgs} /$ mole, and $|\chi|$ decreases with increasing $T$. These results are in agreement with recent experiments [6, 7]. 
We use the nearest-neighbor tight-binding hamiltonian to calculate the band structure formed by the $\pi$ orbitals. This hamiltonian has been shown to be an excellent approximation for calculating the electronic structure of fullerene-related materials such as the large fullerene molecules [9], the solid fullerite and fullerides [10], and the nanotubes [2]. Including the effect of a magnetic field in such a model is straightforward in the London approximation, which has been used successfully for studying the ring current and the magnetic response of $C_{60}$ and $C_{70}$ molecules [11. The symmetry of nanotubes have bees studied by several groups [2]. We follow the elegant approach of White et al. [3]. The structure of a nanotube is defined as the conformal mapping of a strip of a two-dimensional (2-D) graphitic lattice onto the surface of a cylinder. Each nanotube is uniquely characterized by a 2-D lattice vector $\mathbf{L}=n_{1} \mathbf{a}_{1}+n_{2} \mathbf{a}_{2}=\left[n_{1}, n_{2}\right]$, where $\mathbf{a}_{\mathbf{1}}, \mathbf{a}_{\mathbf{2}}$ are 2 -D primitive lattice vectors. The set of integers $\left[n_{1}, n_{2}\right]$ determines the geometric properties of the nanotube. The radius of the tube is $R=\frac{L}{2 \pi}=\frac{\sqrt{3} d_{0}}{2 \pi} \sqrt{n_{1}^{2}+n_{2}^{2}+n_{1} n_{2}}$, where $d_{0}$ is the C-C bond length. There are two symmetry operations: $\mathcal{C}_{N}$ and $\mathcal{S}(\alpha, h) \cdot \mathcal{C}_{N}$ is a $N$-fold rotation along the axis, where $N$ is the largest common denominator of $n_{1}$ and $n_{2}$. The screw translation $\mathcal{S}(h, \alpha)$ represents a rotation of $\alpha$ about the axis followed by a translation of $h$ along the axis. The parameters $h$ and $\alpha$ are determined from a 2-D lattice vector $\mathbf{P}=p_{1} \mathbf{a}_{1}+p_{2} \mathbf{a}_{2}$, where $p_{1}, p_{2}$ are integers which satisfy the condition $p_{2} n_{1}-p_{1} n_{2}=N$ [3]. In the London approximation, the hopping between site $i$ and site $j$ is modified by a phase factor due to the presence of a magnetic field, $V_{i j}=V_{0} \exp \left(i \frac{2 \pi}{\phi_{0}} \int_{i}^{j} \mathbf{A}(\mathbf{r}) \cdot d \mathbf{r}\right)$. Here $V_{0}$ is the nearest-neighbor hopping amplitude, $\mathbf{A}(\mathbf{r})$ is the vector potential associated with the magnetic field, and $\phi_{0}=\frac{h c}{e}$ is the flux quantum.

For the special case of a uniform magnetic field $\mathbf{H}$ parallel to the tube axis $\hat{\mathbf{z}}$, both $\mathcal{C}_{N}$ and $\mathcal{S}(\alpha, h)$ remain symmetry operations of the tube. In this case the hamiltonian can be solved analytically and we obtain the following band structure,

$$
\begin{gathered}
\epsilon_{n}(\kappa)= \pm V_{0}\left[3+2 \cos \left(\delta_{1}\right)+2 \cos \left(\delta_{2}\right)+2 \cos \left(\delta_{1}+\delta_{2}\right)\right]^{1 / 2}, \\
n=0,1, \cdots, N-1, \\
\delta_{1}=\frac{n_{1} \kappa-2 \pi n p_{1}}{N}+\beta\left(n_{1}+2 n_{2}\right),
\end{gathered}
$$




$$
\begin{aligned}
\delta_{2} & =\frac{n_{2} \kappa-2 \pi n p_{2}}{N}-\beta\left(n_{2}+2 n_{1}\right), \\
\beta & =\frac{3 d_{0}^{2} H}{4 \phi_{0}} .
\end{aligned}
$$

Here $\kappa$ is the pseudo-momentum associate with the screw translation $\mathcal{S}(\alpha, h)$. For $H=0$ we recover the result of White et al. [3].

For an idea nanotube each $\pi$ orbital contributes one electron, the Fermi energy is at the center of the band, $\epsilon_{F}=0$. From Eq.(1) one finds that, in addition to the dependence on the helicity and the radius of the nanotube, the band gap $\Delta=2 \min \left\{\epsilon_{n}(\kappa)\right\}$ varies strongly with the magnetic field. Our calculations show that $\Delta$ is a simple period function of the flux, $\phi=\pi R^{2} H$, with a period of $\phi_{0}$,

$$
\Delta(H)= \begin{cases}\Delta_{0} \frac{3 \phi}{\phi_{0}}, & 0 \leq \phi \leq \phi_{0} / 2 \\ \Delta_{0}\left|3-\frac{3 \phi}{\phi_{0}}\right|, & \phi_{0} / 2 \leq \phi \leq \phi_{0}\end{cases}
$$

for $n_{1}-n_{2}=3 q$, and

$$
\Delta(H)= \begin{cases}\Delta_{0}\left|1-\frac{3 \phi}{\phi_{0}}\right|, & 0 \leq \phi \leq \phi_{0} / 2 \\ \Delta_{0}\left|2-\frac{3 \phi}{\phi_{0}}\right|, & \phi_{0} / 2 \leq \phi \leq \phi_{0}\end{cases}
$$

for $n_{1}-n_{2}=3 q \pm 1$, where

$$
\Delta_{0} \approx \frac{V_{0} d_{0}}{R}
$$

is a characteristic energy scale associated with each nanotube.

From above equations one can draw several conclusions. 1) In the absence of the magnetic field there are two types of nanotubes: those with $n_{1}-n_{2}=3 q$ are metallic and those with $n_{1}-n_{2}=3 q \pm 1$ are semiconducting. 2) In the presence of a magnetic field along the tube axis a gap opens up for the metallic tubes. In contrast, the gap decreases for the semiconducting tubes, reaching zero at one-third of the flux quantum. Therefore a field-induced metalinsulator transition is expected for all nanotubes. 3) The gap scales inversely with the tube radius. Its dependence on the magnetic field is controlled by the characteristic field $H^{*}=\phi_{0} /\left(\pi R^{2}\right)$, which can be small for typical nanotubes. For example, taking $V_{0} \sim 2.6 \mathrm{eV}$, 
$d_{0}=1.43 \AA$, one obtains $\Delta \sim 30 \mathrm{meV}$ for a nanotube of radius $R \sim 12 \mathrm{~nm}$. For such a gap, a magnetic field of $3 \mathrm{~T}$ will reduce the gap to zero. Such a field is well within the reach of most experiments. 4) Because of the sensitive dependence of the band structure on the magnetic field, a large magnetoresistance is expected for all carbon nanotube.

For a uniform magnetic field making an arbitrary angle $\theta$ with the tube axis, neither $\mathcal{C}_{N}$ nor $\mathcal{S}(\alpha, h)$ are symmetry operations of the nanotube. But a translation along the tube axis with the lattice vector $\mathbf{T}=\frac{2 n_{1}+n 2}{N} \mathbf{a}_{1}-\frac{n_{1}+2 n_{2}}{N} \mathbf{a}_{2}$ is still a good symmetry operation. For this case, one can obtain the band structure numerically. It is found that the gap is no longer a simple periodic function of the flux. However, our calculations show that for each family of nanotubes the reduced gap $\Delta(H, \theta) / \Delta_{0}$ is a universal function, when the magnetic field is scaled by $H^{*}$. This universal function is shown in Fig.1 for several field directions and for both types of nanotubes. The universal scaling relation enables one to estimate the gap of any nanotube in an arbitrary magnetic field. From Fig.1 one observes that the effect of field on the band gap is reduced when the field direction deviates from the tube axis. This does not imply that physical properties such as the susceptibility are less affected, as we will show.

The strong field dependence of the band structure suggests a large magnetic susceptibility $\chi$. There are two contributions to $\chi$ : the Pauli term $\chi_{P}$ (due to the electron spin) and the orbital term $\chi_{\text {orb }}$ (due to the change in the band energy). Because of the low density of states near the Fermi energy $\chi_{P}$ is negligible [12], $\chi=\chi_{P}+\chi_{\text {orb }} \approx \chi_{\text {orb }}$. At $T=0, \chi$ can be calculated from the second derivative of the total energy, but at a finite temperature the free energy should be used [13],

$$
\begin{aligned}
\chi & =-\left.\frac{\partial^{2} F(H, T)}{\partial H^{2}}\right|_{H=0}, \\
F(H, T) & =-k_{B} T \sum_{n, \kappa} \ln \left[1+\exp \left(-\frac{\left.\epsilon_{n}(\kappa, H)-\mu\right)}{k_{B} T}\right)\right] .
\end{aligned}
$$

The summation is over the complete band. It is important to include the effect of the magnetic field on the complete band structure because $\chi$ is calculated from the total energy which includes contributions far away from the Fermi level. Thus, a perturbative calculation 
near the Fermi energy gives an inaccurate result in general

In the case of ideal carbon nanotubes at $T=0$, the Fermi energy is at the center of the band $\epsilon_{F}=0$. It is found that for $\mathbf{H} \| \hat{\mathbf{z}}$ the metallic nanotubes $\left(n_{1}-n_{2}=3 q\right)$ are paramagnetic while the semiconducting nanotubes $\left(n_{1}-n_{2}=3 q \pm 1\right)$ are diamagnetic. In contrast, for $\mathbf{H} \| \hat{\mathbf{z}}$ all nanotubes are diamagnetic (Fig.2 and Fig.3). The magnitude of the susceptibility increases linearly with the size of the nanotube regardless whether it is diamagnetic or paramagnetic, $|\chi| \sim R$. In general $\chi$ is a sensitive function of $\theta$. Empirically it is found that $\chi(\theta)=a+b \cos (2 \theta)$ is a good approximation (Fig.2). This unusual dependence on the field direction suggests that magnetic poling is a possible method of aligning nanotubes.

An important result found is that $\chi$ is sensitive to the position of the Fermi energy. A small change in the carrier density (hence the Fermi energy) from the half filling, which is likely in real materials, can lead to a large change in $\chi$. The variation of $\chi$ on $\epsilon_{F}$ depends on the characteristic energy scale $\Delta_{0}$. It is found that $\chi / R$ as a function of $\epsilon_{f} / \Delta_{0}$ is universal for each family of nanotubes. Fig.3 shows examples for both $\mathbf{H} \| \hat{\mathbf{z}}$ and $\mathbf{H} \perp \hat{\mathbf{z}}$. One finds that in the case of a perpendicular field, $\chi$ remains diamagnetic in the vicinity of the half filling, while for a parallel field a small deviation from the half filling changes the susceptibility dramatically. (Notice that for the semiconducting nanotubes, the Fermi energy jumps from $\epsilon_{F}=0$ to $\left|\epsilon_{F}\right|>0.5 \Delta_{0}$ for a small change in the carrier density. For a typical nanotube of radius $R \sim 12 \mathrm{~nm}$, the carrier density at $\epsilon_{F}= \pm \Delta_{0}$ is around $1.0 \pm 2.5 \times 10^{-5}$.)

Finally, in Fig.4 we show the temperature dependence of $\chi$. In all cases the magnitude of $\chi$ decreases with increasing $T$. Again, universal scaling is obtained if the temperature is scaled by $T^{*}=\Delta_{0} / k_{B}$. For a typical nanotube of radius $R \sim 12 \mathrm{~nm}, \Delta \sim 30 \mathrm{meV}$, $T^{*} \sim 330 \mathrm{~K}$

Our calculation provides a qualitative explanation for the unusual transport and magnetic properties observed in recent experiments. The large magnetoresistance observed in nanotube bundles [5] is likely related to the field dependence of the band structure near the the Fermi level. If so, our calculations predict that the magnetoresistance should depend 
on the field direction and be sensitive to the carrier density. The magnetic susceptibility was measured by two groups, one group for randomly oriented carbon nanotubes [7] and the other aligned nanotube bundles [6]. At low temperature unusually large diamagnetic susceptibility is found, $\chi \sim-200$ to $-300 \times 10^{-6} \mathrm{cgs} /$ mole for the magnetic field both perpendicular and parallel to the tube axis. From this data we can estimate that the typical radius of nanotubes in those samples is around $R \sim 7$ to $15 \mathrm{~nm}$, a value close to that reported by experiments. In addition, both experiments found that $|\chi|$ decreases substantially from low temperature to room temperature [14], suggesting a characteristic temperature around $300 K$. From our calculation, this observation corresponds to a typical nanotube size of $R \sim 12 \mathrm{~nm}$, consistent with the above estimate. One discrepancy remains a puzzle. Experimentally, $\chi$ is more diamagnetic when the field is parallel to the tube axis than when it is perpendicular. Our calculation suggests the reverse in most cases (Fig.3). Though it is possible to chose a certain $\epsilon_{F}$ such that our results agree with experiments, we feel that existing experiments are not well enough controlled for such a quantitative comparison. For example, the issue of uniformity of nanotubes and the fact that most of them contain multiple shells should be addressed. Clearly, further experiments, such as the doping dependence, are needed for a quantitative test of the present calculations.

In conclusion, we have shown that novel properties are to be expected for carbon nanotubes in a magnetic field. A field-induced metal-insulator transition is predicted for all pure nanotubes. In general, a large magnetoresistance is expected due to the sensitive dependence of the electronic structure on the external magnetic field. The weak-field magnetic susceptibility is large and increases with the nanotube radius. The susceptibility can be either diamagnetic or paramagnetic depending sensitively on the helicity of the nanotube, the field direction, and the position of the Fermi energy. A characteristic energy $\Delta_{0}$ exists for each nanotube. Universal scaling is found for $\chi$ as a function of the reduced Fermi energy $\epsilon_{F} / \Delta_{0}$ and the reduced temperature $k_{B} T / \Delta_{0}$. Both the magnitude and the temperature dependence of the susceptibility are in agreement with recent experiments. Using the current experimental data, it is estimated that typical nanotube radii are around $R \sim 7-15 \mathrm{~nm}$, consistent 
with direct observations. Our results indicate that careful measurements of the magnetic susceptibility provides an efficient probe to characterize nanotubes. The directional dependence of the magnetic response suggests the magnetic poling as a possible method of aligning nanotubes. The novel and unusual magnetic properties of nanotubes may have promising applications in areas such as the magneto-electronics and the magnetic field detectors.

\section{ACKNOWLEDGMENTS}

The author thanks O. Zhou for communicating their experimental results prior to the

publication. This work is supported by The Petroleum Research Fund and the University of North Carolina at Chapel Hill. 


\section{REFERENCES}

[1] S. Iijima, Nature 354, 56 (1991). T. W. Ebbesen and P. M. Ajayan, Nature 358, 220 (1992).

[2] N. Hamada et al., Phys. Rev. Lett. 68, 1579 (1992); L. Wang et al., Phys. Rev. B 46, 7175 (1992); Saito et at., ibid 46, 1804 (1992); M. F. Lin and K. W. Shung, ibid 47, 6617 (1993); R.A. Jishi et al., ibid 48, 11385 (1993).

[3] For the details of the nanotube geometry and notations used in this paper see C. T. White et al., Phys. Rev. B 47, 5485 (1993).

[4] J. W. Mintmire et al, Phys. Rev. Lett. 68, 631 (1992); J. Y. Yi and J. Bernholc, Phys. Rev. B 47, 1708 (1993); X. Blase et al., Phys. Rev. Lett. 72, 1878 (1994).

[5] L. Langer et al., J. Mater. Res. 9, 927 (1994); S.N. Song et al., Phys. Rev. Lett. 72, 697 (1994).

[6] X.K. Wang et al., J. Mater. Res. 9, 1578 (1994).

[7] A. P. Ramirez et al., Science 265, 84 (1994).

[8] H. Ajiki and T. Ando, J. Phys. Soc. Jpn. 62, 1255 (1993); 62, 2470 (1993).

[9] J.P. Lu and W. Yang, Phys. Rev. B 49, 11421 (1994).

[10] M. Gelfand and J.P. Lu, Phys. Rev. Lett. 68, 1050 (1992); Phys. Rev. B 46, 4367 (1992); S. Satpathy et al., ibid 46, 1773 (1992).

[11] V. Elser and R.C. Haddon, Nature 325, 792 (1987); A. Pasquarello et al., Phys. Rev. B 47, 1783 (1993); M. Saunders et al., Nature 367, 256 (1994).

[12] $\chi_{P}$ measures the density of states at the Fermi level, $\chi_{P} \sim 1 / R$. For $R \sim 10 \mathrm{~nm}, \chi_{P}$ is three orders of magnitude smaller than $\chi_{\text {orb }}$.

[13] In the case of graphite this makes a factor of three difference in $\chi$ at room temperature. 
See J.W. McClure, Phys. Rev. 104, 666 (1956).

[14] Part of the residual susceptibility observed at the room temperature may be related to the sample quality, as its value is close to the susceptibility of the polycrystalline graphite. This factor may also affect the relative magnitude of $\chi_{\|}$and $\chi_{\perp}$. 


\section{FIGURES}

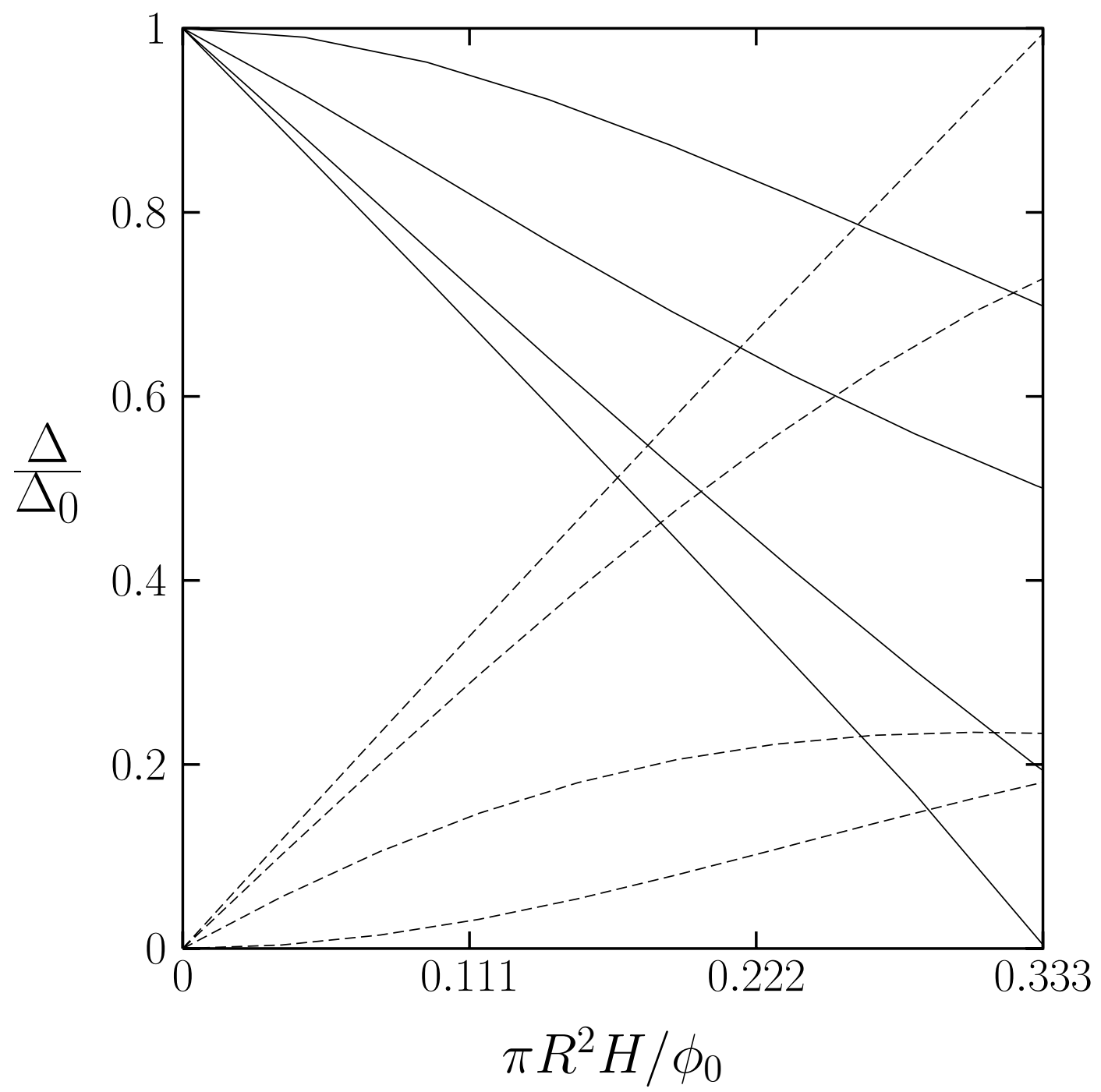

FIG. 1. The reduced gap $\Delta / \Delta_{0}$ as a function of the magnetic field $H$ for different field directions. Solid lines: semiconducting tubes $\left(n_{1}-n_{2}=3 q \pm 1\right.$ ), $\theta=\pi / 2, \pi / 3, \pi / 6,0$ (top down). Dashed lines: metallic tubes $\left(n_{1}-n_{2}=3 q\right), \theta=\pi / 2, \pi / 3, \pi / 6,0$ (bottom up). $\theta$ is the angle between the field direction and the tube axis, $\Delta_{0}=V_{0} d_{0} / R$. For all figures presented in this paper we use $V_{0}=2.6 \mathrm{eV}, d_{0}=1.43 \AA$. 


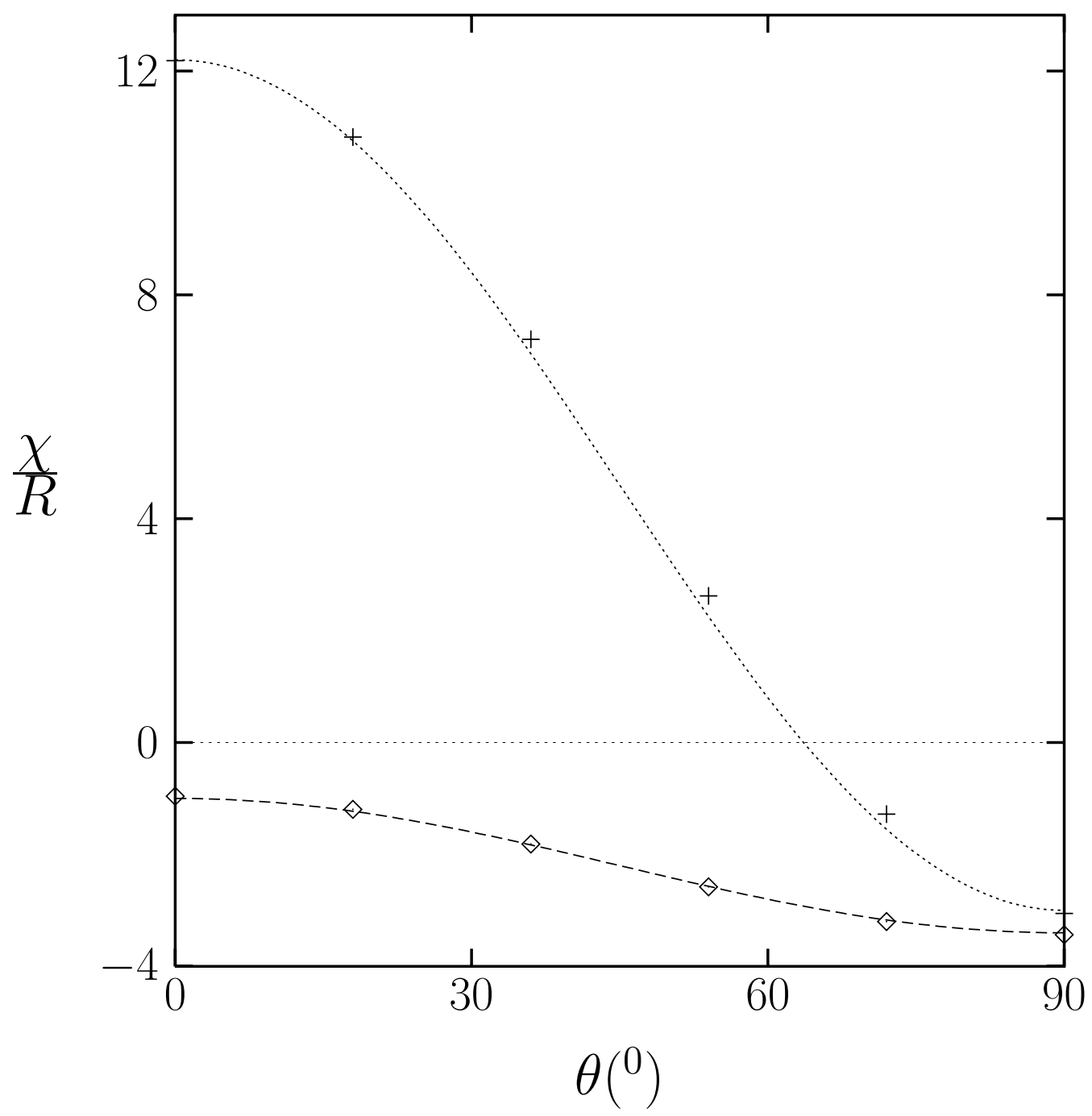

FIG. 2. The scaled susceptibility $\chi / R$ as a functions of the angle $\theta$ between the magnetic field direction and the tube axis. $\epsilon_{F}=0, T=0$. Plus: $n_{1}-n_{2}=3$. Diamonds: $n_{1}-n_{2}=3 q+1$. Lines are fits to the functional form $a+b \cos (2 \theta)$. The case of $n_{1}-n_{2}=3 q-1$ is very similar to that of $n_{1}-n_{2}=3 q+1 . \chi$ is in units of $10^{-6} \mathrm{cgs} /$ mole, $R$ is in units of $\AA$. 


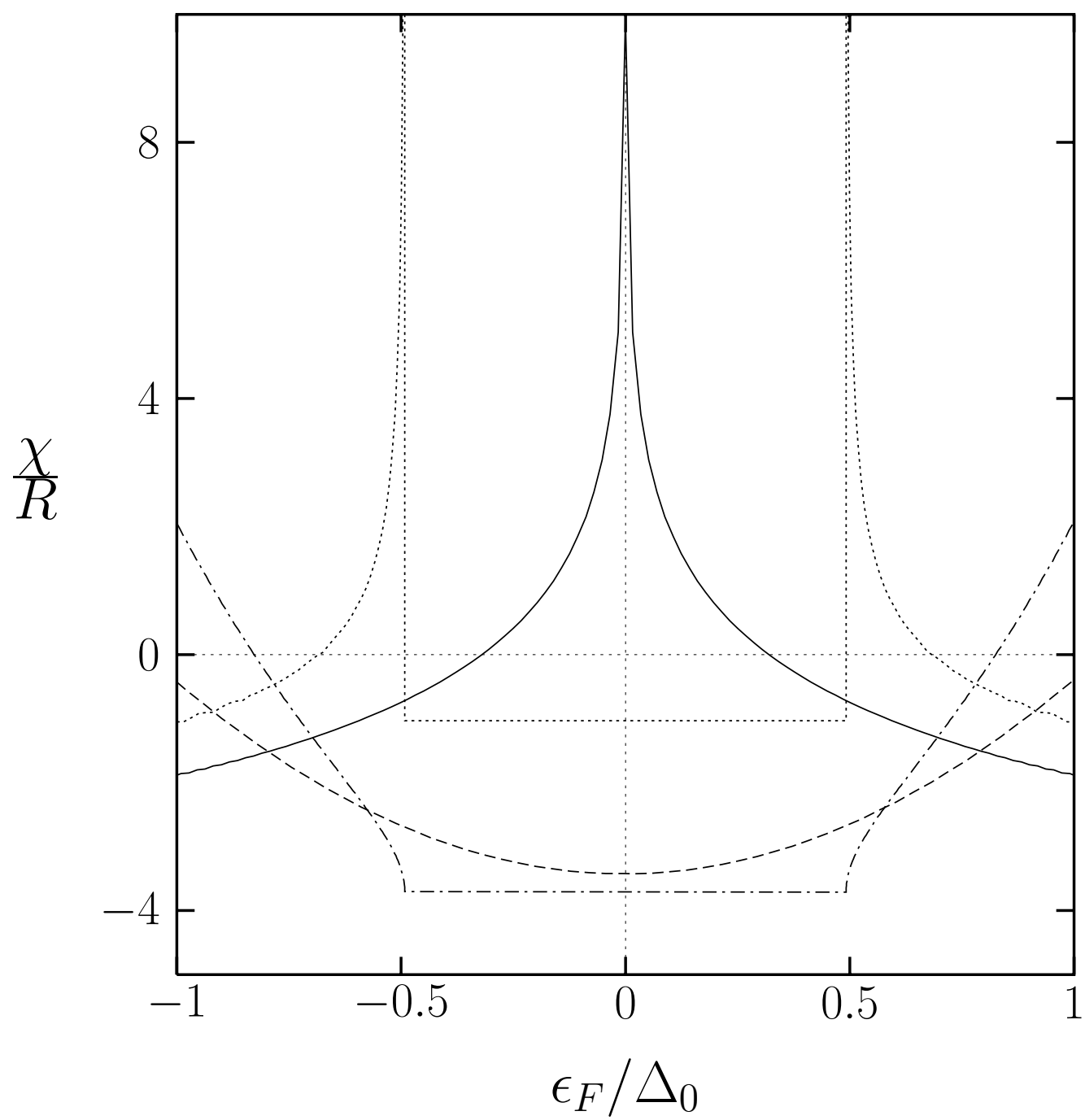

FIG. 3. Universal scaling of $\chi / R$ as a function of the reduced Fermi energy $\epsilon_{F} / \Delta_{0} . T=0$. Solid line: $\mathbf{H} \| \hat{\mathbf{z}}, n_{1}-n_{2}=3 q$. Dotted line: $\mathbf{H} \| \hat{\mathbf{z}}, n_{1}-n_{2}=3 q+1$. Dashed line: $\mathbf{H} \perp \hat{\mathbf{z}}, n_{1}-n_{2}=3 q$. Dot-Dashed line: $\mathbf{H} \perp \hat{\mathbf{z}}, n_{1}-n_{2}=3 q+1$. The case of $n_{1}-n_{2}=3 q-1$ is very similar to that of $n_{1}-n_{2}=3 q+1 . \chi / R$ is in units of $10^{-6} \mathrm{cgs} / \mathrm{mole} / \AA$. 


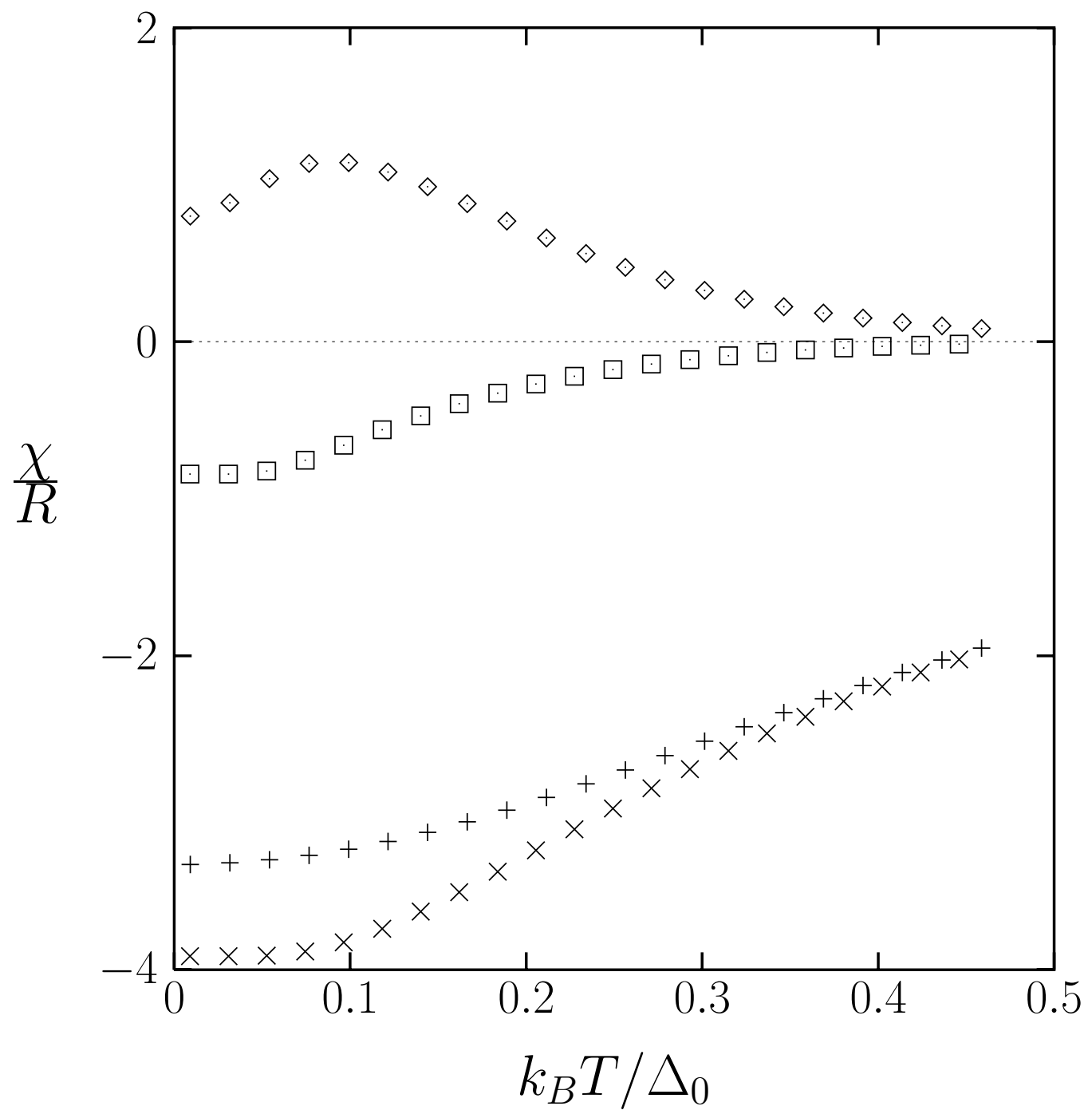

FIG. 4. The universal dependence of the scaled susceptibility $\chi / R$ on the reduced temperature $k_{B} T / \Delta_{0}$. The Fermi energy is at $\epsilon_{F}=0.2 \Delta_{0}$. Squares: $\mathbf{H} \| \hat{\mathbf{z}}, n_{1}-n_{2}=3 q$. Diamonds: $\mathbf{H} \| \hat{\mathbf{z}}, n_{1}-n_{2}=3 q-1$. Plus: $\mathbf{H} \perp \hat{\mathbf{z}}, n_{1}-n_{2}=3 q$. Crosses: $\mathbf{H} \perp \hat{\mathbf{z}}, n_{1}-n_{2}=3 q-1$. The case of $n_{1}-n_{2}=3 q+1$ is very similar to that of $n_{1}-n_{2}=3 q-1 . \chi / R$ is in units of $10^{-6} \mathrm{cgs} / \mathrm{mole} / \AA$. 